\title{
ABSOLUTELY CONTINUOUS SPECTRUM IN THE ANDERSON MODEL ON THE BETHE LATTICE
}

\begin{abstract}
ABEL KLEin
Abstract. We prove that the spectrum of the Anderson Hamiltonian $H_{\lambda}=-\Delta+\lambda V$ on the Bethe Lattice is absolutely continuous inside the spectrum of the Laplacian, if the disorder $\lambda$ is sufficiently small. More precisely, given any closed interval $I$ contained in the interior of the spectrum of the (centered) Laplacian $\Delta$ on the Bethe lattice, we prove that for small disorder, $H_{\lambda}$ has purely absolutely continuous spectrum in $I$ with probability one (i.e., $\sigma_{a c}\left(H_{\lambda}\right) \cap I=I$ and $\sigma_{p p}\left(H_{\lambda}\right) \cap I=\sigma_{s c}\left(H_{\lambda}\right) \cap I=\emptyset$ with probability one).
\end{abstract}

The Bethe lattice (or Cayley tree), $\mathbb{B}$, is an infinite connected graph with no closed loops and a fixed degree (number of nearest neighbors) at each vertex (site or point). The degree is called the coordination number and the connectivity, $K$, is one less the coordination number. The distance between two sites $x$ and $y$ will be denoted by $d(x, y)$ and is equal to the length of the shortest path connecting $x$ and $y$.

The Anderson model on the Bethe lattice is given by the random Hamiltonian

$$
H_{\lambda}=\frac{1}{2} \Delta+\lambda V
$$

on

$$
\ell^{2}(\mathbb{B})=\left\{u: \mathbb{B} \rightarrow \mathbb{C} ; \sum_{x \in \mathbb{B}}|u(x)|^{2}<\infty\right\} .
$$

The (centered) Laplacian $\Delta$ is defined by

$$
(\Delta u)(x)=\sum_{y: d(x, y)=1} u(y) ;
$$

it has spectrum $\sigma(\Delta)=[-2 \sqrt{K}, 2 \sqrt{K}]$ (e.g., [3]); we use $\frac{1}{2} \Delta$ in the definition of $H_{\lambda}$ to simplify some formulas (notice that in the Bethe lattice $-\Delta$ and $\Delta$ are unitarily equivalent). $V$ is a random potential, with $V(x)$,

Received May 4, 1994.

1991 Mathematics Subject Classification. Primary 82B44. Secondary 81Q10.

Key words and phrases: random Schrodinger operators, Anderson model, extended states, absolutely continuous spectrum, localization.

Partially supported by NSF grant DMS-9208029. 
$x \in \mathbb{B}$, being independent, identically distributed random variables with common probability distribution $\mu$. The characteristic function of $\mu$ will be denoted by $h$, i.e., $h(t)=\int e^{-i t v} d \mu(v)$. The real parameter $\lambda$ is called the disorder.

In this announcement we always assume that $K \geq 2$ (so $\mathbb{B}$ is not the line $\mathbb{R})$ and that $h(t)$ is differentiable on $(0, \infty)$, with $h^{\prime}(t)$ absolutely continuous and bounded on $(0, \infty)$, and $h^{\prime \prime}(t)$ also bounded. These conditions are satisfied by any probability distribution $\mu$ with a finite second moment (e.g., uniform, Gaussian or Bernoulli distributions) and by the Cauchy distribution.

It follows from ergodicity (the ergodic theorem in the Bethe lattice is discussed in the Appendix of [3]) that the spectrum of the Hamiltonian $H_{\lambda}$ is given by

$$
\sigma\left(H_{\lambda}\right)=\sigma\left(\frac{1}{2} \Delta\right)+\lambda \operatorname{supp} \mu=[-\sqrt{K}, \sqrt{K}]+\lambda \operatorname{supp} \mu
$$

with probability one $[25,8]$. For each choice of $V$, the spectrum of $H_{\lambda}$ can be decomposed into pure point spectrum, $\sigma_{p p}\left(H_{\lambda}\right)$; absolutely continuous spectrum, $\sigma_{a c}\left(H_{\lambda}\right)$; and singular continuous spectrum, $\sigma_{s c}\left(H_{\lambda}\right)$. Ergodicity gives the existence of sets $\Sigma_{\lambda, p p}, \Sigma_{\lambda, a c}, \Sigma_{\lambda, s c} \subset \mathbb{R}$ such that $\sigma_{p p}\left(H_{\lambda}\right)=\Sigma_{\lambda, p p}, \sigma_{a c}\left(H_{\lambda}\right)=\Sigma_{\lambda, a c}$ and $\sigma_{s c}\left(H_{\lambda}\right)=\Sigma_{\lambda, s c}$ with probability one $[20,8]$.

The main result in this announcement is a proof of the existence of absolutely continuous spectrum in the Anderson model.

Theorem 1. For any $E, 0<E<\sqrt{K}$, there exists $\lambda(E)>0$, such that for any $\lambda$ with $|\lambda|<\lambda(E)$, the spectrum of $H_{\lambda}$ in $[-E, E]$ is purely absolutely continuous with probability one, i.e., we have $\Sigma_{\lambda, a c} \cap[-E, E]=$ $[-E, E]$ and $\Sigma_{\lambda, p p} \cap[-E, E]=\Sigma_{\lambda, s c} \cap[-E, E]=\emptyset$.

Anderson [7] introduced this model to describe the motion of a quantummechanical electron in a crystal with impurities. In one and two dimensions it was argued that, as long as the potential was random (i.e., $\lambda \neq 0$ ), the model exhibits exponential localization (i.e., pure point spectrum with exponentially decaying eigenfunctions). In three and more dimensions both localized and extended states (i.e., absolutely continuous spectrum) are expected for small disorder, with the energies of extended and localized states being separated by the "mobility edge". A new approach to the study of such questions was given by Abou-Chacra, Anderson and Thouless [1] and Abou-Chacra and Thouless [2], who developed a self-consistent approximation for the study of localization which becomes exact in the Bethe lattice, where they argued that there should be a mobility edge for small 
disorder. They calculated that the energy at which localization breaks down converges to $\frac{K+1}{2}$ in the zero disorder limit.

The Physics literature contains many papers which study the Anderson model in the Bethe lattice; the most recent ones being the work of Mirlin and Fyodorov [23] and of Miller and Derrida [22]; we refer to their list of references for other related work. Miller and Derrida performed a weak disorder expansion inside the spectrum of the zero disorder Hamiltonian, and computed perturbatively the density of states and conducting properties corresponding to extended states. They also found the existence of an energy, which converges to the edge $\sqrt{K}$ of the spectrum of $\frac{1}{2} \Delta$ in the zero disorder limit, above which the density of states and the conducting properties vanish to all orders in perturbation theory.

Localization for the Anderson Hamiltonian is by now well understood. In one dimension there are mathematical proofs of exponential localization for any disorder (e.g., [15, 21, 9, 12] and others). In the multidimensional case exponential localization is proved for large disorder or low energy (e.g., $[14,13,11,26,12,6,4,17]$ and others). Only localization in two dimensions for small disorder is still an open problem.

But up to now there was no proof of the occurrence of absolutely continuous spectrum (i.e., extended states) in the Anderson model.

For the Bethe lattice there were no rigorous results up to recently. Kunz and Souillard [21] gave an outline of what should be proven: analyticity of the density of states for distributions close to the Cauchy distribution, localization for large disorder or low energies, and existence of extended states for small disorder. The first was proved by Acosta and Klein [3], the second by Aizenman and Molchanov [6], and the third is the subject of this announcement.

Aizenman [4] has proved localization in the Bethe lattice for energies beyond $\frac{K+1}{2}$ at weak disorder, confirming half of Abou-Chacra and Thouless' prediction [2]. We prove purely absolutely continuous spectrum below $\sqrt{K}$ at weak disorder, in agreement with Miller and Derrida [22]. This leaves open the question of the nature of the spectrum for energies $E$ with $\sqrt{K} \leq|E| \leq \frac{K+1}{2}$.

For the case when the potential at a single site has a Cauchy distribution, Aizenman [5] has announced a proof of the existence of extended states (absolutely continuous spectrum), inside the spectrum of $\frac{1}{2} \Delta$, for small disorder.

The Green's function of $H_{\lambda}$ is given by

$$
G_{\lambda}(x, y ; z)=\left\langle x\left|\left(H_{\lambda}-z\right)^{-1}\right| y\right\rangle
$$

for $x, y \in \mathbb{B}$ and $z=E+i \eta$ with $E \in \mathbb{R}, \eta>0$. For any $x \in \mathbb{B}$ and 
any potential $V, G_{\lambda}(x, x ; E+i \eta)$ is a continuous function of $(\lambda, E, \eta) \in$ $\mathbb{R} \times \mathbb{R} \times(0, \infty)$, so $\mathbb{E}\left(G_{\lambda}(x, x ; E+i \eta)\right)$ and $\mathbb{E}\left(\left|G_{\lambda}(x, x ; E+i \eta)\right|^{2}\right)$ are also continuous functions of $(\lambda, E, \eta) \in \mathbb{R} \times \mathbb{R} \times(0, \infty)$. Theorem 1 will follow from the fact that we can let $\eta \downarrow 0$ inside the spectrum of $\frac{1}{2} \Delta$.

Theorem 2. For any $E, 0<E<\sqrt{K}$, there exists $\lambda(E)>0$, such that for all $x \in \mathbb{B}$ the continuous function

$$
\begin{aligned}
\left(\lambda, E^{\prime}, \eta\right) \in(-\lambda(E), \lambda(E)) \times[-E, E] & \times(0, \infty) \\
& \longrightarrow \mathbb{E}\left(\left|G_{\lambda}\left(x, x ; E^{\prime}+i \eta\right)\right|^{2}\right)
\end{aligned}
$$

has a continuous extension to $(-\lambda(E), \lambda(E)) \times[-E, E] \times[0, \infty)$. In particular,

$$
\sup _{\lambda ;|\lambda|<\lambda(E)} \sup _{E^{\prime} ;\left|E^{\prime}\right| \leq E} \sup _{\eta ; 0<\eta} \mathbb{E}\left(\left|G_{\lambda}\left(x, x ; E^{\prime}+i \eta\right)\right|^{2}\right)<\infty .
$$

Theorem 1 follows from (5) by the following criterion for the absolute continuity of a measure in terms of its Stieltjes transform:

Theorem 3. Let $\nu$ be a finite measure on $\mathbb{R}$, and for $a>0$, set $\nu_{a}$ to be the restriction of $\nu$ to the interval $(-a, a)$. Suppose

$$
\liminf _{\eta \downarrow 0} \int_{-a}^{a}|F(E+i \eta)|^{2} d E<\infty
$$

where $F(z)=\int \frac{d \nu(t)}{t-z}, z=E+i \eta$ with $\eta>0$, is the Stieltjes (or Borel) transform of $\nu$. Then $\nu_{a}$ is absolutely continuous.

We will now sketch the proof of Theorem 2. We fix an arbitrary site in $\mathbb{B}$ which we will call the origin and denote by 0 , and let $\mathbb{B}^{(0)}$ be the lattice obtained by removing from $\mathbb{B}$ one branch emanating from 0 . Each vertex in $\mathbb{B}^{(0)}$ has degree $K+1$, with the single exception of the origin 0 which has degree $K$. We will use $G_{\lambda}^{(0)}(x, y ; z)$ to denote the Green's function of the operator $H_{\lambda}$ restricted to $\mathbb{B}^{(0)}$ with Dirichlet boundary conditions. In addition, we will write $G_{\lambda}(z)$ and $G_{\lambda}^{(0)}(z)$ for $G_{\lambda}(0,0 ; z)$ and $G_{\lambda}^{(0)}(0,0 ; z)$, respectively.

Lemma 4. For any $\lambda \in \mathbb{R}, E \in \mathbb{R}$ and $\eta>0$ let

$$
\begin{aligned}
& \xi_{\lambda, z}\left(\varphi_{+}^{2}, \varphi_{-}^{2}\right)=\mathbb{E}\left(\exp \left\{\frac{i}{4}\left(G_{\lambda}^{(0)}(z) \varphi_{+}^{2}-\overline{G_{\lambda}^{(0)}(z)} \varphi_{-}^{2}\right)\right\}\right) \\
& =\mathbb{E}\left(\exp \left\{\frac{1}{4}\left[i \mathcal{R}_{\lambda}^{(0)}(z)\left(\varphi_{+}^{2}-\varphi_{-}^{2}\right)-\mathcal{I}_{\lambda}^{(0)}(z)\left(\varphi_{+}^{2}+\varphi_{-}^{2}\right)\right]\right\}\right),
\end{aligned}
$$


where $\mathcal{R}_{\lambda}^{(x \mid 0)}(z)+i \mathcal{I}_{\lambda}^{(x \mid 0)}(z)$ is the decomposition of $G_{\lambda}^{(x \mid 0)}(z)$ into its real and imaginary parts. Then

$$
\begin{aligned}
\mathbb{E}\left(\left|G_{\lambda}(z)\right|^{2}\right)=\frac{1}{\pi^{2}} \int_{\mathbb{R}^{2} \times \mathbb{R}^{2}} e^{i E\left(\varphi_{+}^{2}-\varphi_{-}^{2}\right)-\eta\left(\varphi_{+}^{2}+\varphi_{-}^{2}\right)} & \\
& h\left(\lambda\left(\varphi_{+}^{2}-\varphi_{-}^{2}\right)\right)\left[\xi_{\lambda, z}\left(\varphi_{+}^{2}, \varphi_{-}^{2}\right)\right]^{K+1} d^{2} \varphi_{+} d^{2} \varphi_{-},
\end{aligned}
$$

and

$$
\begin{aligned}
& \xi_{\lambda, z}\left(\varphi_{+}^{2}, \varphi_{-}^{2}\right)=\frac{1}{\pi^{2}} \int_{\mathbb{R}^{2} \times \mathbb{R}^{2}} e^{-i\left(\varphi_{+} \cdot \varphi_{+}^{\prime}-i \varphi_{-} \cdot \varphi_{-}^{\prime}\right)} \\
& \partial_{+} \partial_{-}\left\{e^{i E\left(\varphi_{+}^{\prime}{ }^{2}-\varphi_{-}^{\prime}{ }^{2}\right)-\eta\left(\varphi_{+}^{\prime}{ }^{2}+\varphi_{-}^{\prime}{ }^{2}\right)} h\left(\lambda\left(\varphi_{+}^{\prime}{ }^{2}-\varphi_{-}^{\prime}{ }^{2}\right)\right)\left[\xi_{\lambda, z}\left(\varphi_{+}^{\prime}{ }^{2}, \varphi_{-}^{\prime}{ }^{2}\right)\right]^{K}\right\} \\
& d^{2} \varphi_{+}^{\prime} d^{2} \varphi_{-}^{\prime},
\end{aligned}
$$

with

$$
\partial_{ \pm} g\left(\varphi_{+}^{2}, \varphi_{-}^{2}\right)=\frac{\partial}{\partial \varphi_{ \pm}^{2}} g\left(\varphi_{+}^{2}, \varphi_{-}^{2}\right)
$$

The simplest way to prove Lemma 4 is to undo the expectations in (9) and (10) and perform the integration on the right-hand sides; the resulting equations can be verified by an argument based on the resolvent identity. A more intuitive derivation uses the "supersymmetric replica trick" [16].

If $\lambda=0$ we can calculate $G_{0}^{(0)}(z)$ obtaining [3]

$$
\xi_{0, z}\left(\varphi_{+}^{2}, \varphi_{-}^{2}\right)=e^{\frac{i}{2 K}\left\{\left(-z+\sqrt{z^{2}-K}\right) \varphi_{+}^{2}-\overline{\left(-z+\sqrt{z^{2}-K}\right)} \varphi_{-}^{2}\right\}}
$$

where we always make the choice $\operatorname{Im} \sqrt{ }>0$. If $|E|<\sqrt{K}$, we have the pointwise limit

$$
\begin{aligned}
\xi_{0, E}\left(\varphi_{+}^{2}, \varphi_{-}^{2}\right) & \equiv \lim _{\eta \downarrow 0} \xi_{0, z}\left(\varphi_{+}^{2}, \varphi_{-}^{2}\right) \\
& =e^{\frac{1}{2 K}\left\{-i E\left(\varphi_{+}^{2}-\varphi_{-}^{2}\right)-\sqrt{K-E^{2}}\left(\varphi_{+}^{2}+\varphi_{-}^{2}\right)\right\}} .
\end{aligned}
$$

To handle the nonlinear equations (9) and (10), we introduce the Banach spaces $\mathcal{K}_{p}, 1 \leq p \leq \infty$, given by the completion of

$$
\begin{aligned}
\left\{g:[0, \infty) \times[0, \infty) \rightarrow \mathbb{C} \text { of class } C^{2} ;\right. \\
\left.\|g\|_{\mathcal{K}} \equiv\|\|\left\|_{2}+\right\| g \|_{p}<\infty\right\}
\end{aligned}
$$


where

$$
\begin{aligned}
\|\| g\left\|_{p}^{2}=\right\| & \left(\varphi_{+}^{2}, \varphi_{-}^{2}\right) \|_{L^{p}\left(\mathbb{R}^{2} \times \mathbb{R}^{2}, d^{2} \varphi_{+} d^{2} \varphi_{-}\right)}^{2} \\
& +\left\|2 \partial_{+} g\left(\varphi_{+}^{2}, \varphi_{-}^{2}\right)\right\|_{L^{p}\left(\mathbb{R}^{2} \times \mathbb{R}^{2}, d^{2} \varphi_{+} d^{2} \varphi_{-}\right)}^{2} \\
& +\left\|2 \partial_{-} g\left(\varphi_{+}^{2}, \varphi_{-}^{2}\right)\right\|_{L^{p}\left(\mathbb{R}^{2} \times \mathbb{R}^{2}, d^{2} \varphi_{+} d^{2} \varphi_{-}\right)}^{2} \\
& +\left\|4 \partial_{+} \partial_{-} g\left(\varphi_{+}^{2}, \varphi_{-}^{2}\right)\right\|_{L^{p}\left(\mathbb{R}^{2} \times \mathbb{R}^{2}, d^{2} \varphi_{+} d^{2} \varphi_{-}\right)}^{2} .
\end{aligned}
$$

We define the linear operators

$$
\begin{aligned}
& (\mathcal{T} g)\left(\varphi_{+}^{2}, \varphi_{-}^{2}\right) \\
& =\frac{1}{\pi^{2}} \int_{\mathbb{R}^{2} \times \mathbb{R}^{2}} e^{-i\left(\varphi_{+} \cdot \varphi_{+}^{\prime}-i \varphi_{-} \cdot \varphi_{-}^{\prime}\right)} \partial_{+} \partial_{-}\left\{g\left(\varphi_{+}^{\prime}{ }^{2}, \varphi_{-}^{\prime}{ }^{2}\right)\right\} d^{2} \varphi_{+}^{\prime} d^{2} \varphi_{-}^{\prime}
\end{aligned}
$$

and

$$
\mathcal{B}(\lambda, z)=M\left(e^{i E\left(\varphi_{+}^{2}-\varphi_{-}^{2}\right)-\eta\left(\varphi_{+}^{2}+\varphi_{-}^{2}\right)} h\left(\lambda\left(\varphi_{+}^{2}-\varphi_{-}^{2}\right)\right)\right),
$$

where $M\left(g\left(\varphi_{+}^{2}, \varphi_{-}^{2}\right)\right)$ denotes multiplication by the function $g\left(\varphi_{+}^{2}, \varphi_{-}^{2}\right)$.

It turns out that $\mathcal{T}$ is unitary on $\mathcal{K}_{2}$ [8] and is a bounded linear operator from $\mathcal{K}_{1}$ to $\mathcal{K}_{\infty}, \mathcal{B}_{\lambda, z}$ is a bounded linear operator on all $\mathcal{K}_{p}$, and $g \rightarrow g^{n}$ is a continuous map from $\mathcal{K}_{\infty}$ to $\mathcal{K}_{1}$ for any $n=2,3, \ldots$ Moreover,

\section{Lemma 5.}

(I). $\xi_{\lambda, z} \in \mathcal{K}_{\infty}$ for all $\lambda \in \mathbb{R}$ and $z=E+i \eta$ with $\eta>0$. The map $(\lambda, E, \eta) \rightarrow \xi_{\lambda, E+i \eta}$ is continuous from $\mathbb{R} \times \mathbb{R} \times(0, \infty)$ to $\mathcal{K}_{\infty}$.

(II). If $|E|<\sqrt{K}$ we have $\xi_{0, E} \in \mathcal{K}_{\infty}$ and

$$
\lim _{\eta \downarrow 0} \xi_{0, E+i \eta}=\xi_{0, E} \quad \text { in } \mathcal{K}_{\infty} .
$$

(III). (10) can be rewritten as a fixed point equation in $\mathcal{K}_{\infty}$ :

$$
\xi_{\lambda, z}=\mathcal{T} \mathcal{B}_{\lambda, z} \xi_{\lambda, z}^{K},
$$

valid for all $\lambda \in \mathbb{R}$ and $z=E+i \eta$ with $\eta>0$, and also valid for $\lambda=0$ and $z=E$ with $|E|<\sqrt{K}$.

We can now do a fixed point analysis.

Lemma 6. The map $Q: \mathbb{R} \times \mathbb{R} \times[0, \infty) \times \mathcal{K}_{\infty} \rightarrow \mathcal{K}_{\infty}$, defined by

$$
Q(\lambda, E, \eta, g)=\mathcal{T} \mathcal{B}_{\lambda, E+i \eta} g^{K}-g,
$$

is continuous. $Q$ is continuously Frechet differentiable with respect to $g$, the partial derivative being

$$
Q_{g}(\lambda, E, \eta, g)=K \mathcal{T} \mathcal{B}_{\lambda, E+i \eta} M\left(g^{K-1}\right)-I
$$


Moreover, for any $E$ such that $|E|<\sqrt{K}$ we have $Q\left(0, E, 0, \xi_{0, E}\right)=0$ and

$$
0 \notin \sigma\left(Q_{g}\left(0, E, 0, \xi_{0, E}\right)\right) \text {. }
$$

The proof of this lemma is straightforward except for (22). We have $Q_{g}\left(0, E, 0, \xi_{0, E}\right)=K \mathcal{A}_{0, E}-I$ where $\mathcal{A}_{0, E}=\mathcal{T} \mathcal{B}_{0, E} M\left(\xi_{0, E}^{K-1}\right)$. Following Acosta and Klein [3] (see their Propositions 3.2 and 3.3 and Theorem 3.5), we show that $\mathcal{A}_{0, E}^{2}$ is a compact operator on $\mathcal{K}_{\infty}$ and

$$
\sigma\left(\mathcal{A}_{0, E}\right)=\left\{\mathcal{E}_{i, j}=E_{i} \bar{E}_{j} ; i, j=0,1,2, \ldots\right\} \cup\{0\},
$$

with

$$
E_{n}=\left(\frac{-E+i \sqrt{K-E^{2}}}{K}\right)^{2 n} \text { for } n=0,1,2, \ldots
$$

Since $\mathcal{E}_{i, j} \neq \frac{1}{K}$ for any $i, j=0,1,2, \ldots,(22)$ follows.

Lemma 6 tells us that the hypotheses of the Implicit Function Theorem (see 2.7.2 in [24]) are satisfied by the function $Q(\lambda, E, \eta, g)$ at $\left(0, E, 0, \xi_{0, E}\right)$, if $|E|<\sqrt{K}$. It follows that for each $E$ such that $|E|<\sqrt{K}$, there exist $\lambda_{E}>0, \varepsilon_{E}>0, \eta_{E}>0$ and $\delta_{E}>0$, such that for each

$$
\left(\lambda, E^{\prime}, \eta\right) \in\left(-\lambda_{E}, \lambda_{E}\right) \times\left(E-\varepsilon_{E}, E+\varepsilon_{E}\right) \times\left[0, \eta_{E}\right)
$$

there is a unique $\omega_{\lambda, E^{\prime}, \eta} \in \mathcal{K}_{\infty}$ with $\left\|\omega_{\lambda, E^{\prime}, \eta}-\xi_{0, E}\right\|_{\mathcal{K}_{\infty}}<\delta_{E}$, such that $Q\left(\lambda, E^{\prime}, \eta, \omega_{\lambda, E^{\prime}, \eta}\right)=0$. Moreover, the map

$$
\left(\lambda, E^{\prime}, \eta\right) \in\left(-\lambda_{E}, \lambda_{E}\right) \times\left(E-\varepsilon_{E}, E+\varepsilon_{E}\right) \times\left[0, \eta_{E}\right) \longrightarrow \omega_{\lambda, E^{\prime}, \eta} \in \mathcal{K}_{\infty}
$$

is continuous. Combining with Lemma 5, and using the uniqueness of $\omega_{\lambda, E^{\prime}, \eta}$ as above for each $\left(\lambda, E^{\prime}, \eta\right)$, we get

Theorem 7. For any $E$ such that $|E|<\sqrt{K}$, there exist $\lambda_{E}>0, \varepsilon_{E}>0$ and $\delta_{E}>0$, such that the map

$$
\begin{aligned}
\left(\lambda, E^{\prime}, \eta\right) \in\left(-\lambda_{E}, \lambda_{E}\right) \times\left(E-\varepsilon_{E}, E+\varepsilon_{E}\right) \times & (0, \infty) \\
& \longrightarrow \xi_{\lambda, E^{\prime}+i \eta} \in \mathcal{K}_{\infty}
\end{aligned}
$$

has a continuous extension to

$$
\left(-\lambda_{E}, \lambda_{E}\right) \times\left(E-\varepsilon_{E}, E+\varepsilon_{E}\right) \times[0, \infty)
$$

satisfying (19).

Theorem 2 now follows from (9), Theorem 7, the translation invariance of expectations, and a simple compactness argument.

Full details can be found in Klein [18]. 


\section{References}

1. R. Abou-Chacra, P. Anderson and D. J. Thouless, A self-consistent theory of localization, J. Phys. C: Solid State Phys. 6 (1973), 1734-1752.

2. P. Abou-Chacra and D. J. Thoulesszz Self-consistent theory of localization: II. Localization near the band edges, J. Phys. C: Solid State Phys. 7 (1974), 65-75.

3. V. Acosta and A. Klein, Analyticity of the density of states in the Anderson model in the Bethe lattice, J. Stat. Phys. 69 (1992), 277-305.

4. M. Aizenman, Localization at weak disorder: some elementary bounds, Rev. Math. Phys., to appear.

5.

6. M. Aizenman and S. Molchanov, Localization at large disorder and extreme energies: an elementary derivation, Commun. Math. Phys., to appear.

7. P. Anderson, Absence of diffusion in certain random lattices, Phys. Rev. 109 (1958), 1492-1505.

8. M. Campanino and A. Klein, A supersymmetric transfer matrix and differentiability of the density of states in the one-dimensional Anderson model, Comm. Math. Phys. 104 (1986), 227-241.

9. R. Carmona, A. Klein and F. Martinelli, Anderson localization for Bernoulli and other singular potentials, Commun. Math. Phys. 108 (1987), 41-66.

10. R. Carmona and J. Lacroix, Spectral Theory of Random Schrodinger Operators, Birkhauser, Boston, MA, 1990.

11. F. Delyon, Y. Levy and B. Souillard, Anderson localization for multidimensional systems at large disorder or low energy, Commun. Math. Phys. 100 (1985), 463470.

12. H. von Dreifus and A. Klein, A new proof of localization in the Anderson tight binding model, Commun. Math. Phys. 124 (1989), 285-299.

13. J. Fröhlich, F. Martinelli, E. Scoppola and T. Spencer, Constructive proof of localization in the Anderson tight binding model, Commun. Math. Phys. 101 (1985), 21-46.

14. J. Fröhlich and T. Spencer, Absence of diffusion in the Anderson tight binding model for large disorder or low energy, Commun. Math. Phys. 88 (1983), 151-184.

15. Ya. Gol'dsheid, S. Molchanov and L. Pastur, Pure point spectrum of stochastic one dimensional Schrödinger operators, Funct. Anal. Appl. 11 (1977), 1-10.

16. A. Klein, The supersymmetric replica trick and smoothness of the density of states for random Schrodinger operators, Proc. Symposia in Pure Mathematics 51 (1990), $315-331$.

17. Localization in the Anderson model with long range hopping, Braz. J. Phys. 23 (1993), 363-371.

18. __ Extended states in the Anderson model on the Bethe lattice, submitted for publication.

19. A. Klein and A. Speis, Regularity of the invariant measure and of the density of states in the one-dimensional Anderson model, J. Funct. Anal. 88 (1990), 211-227.

20. H. Kunz and B. Souillard, Sur le spectre des operateurs aux differences finies aleatoires, Commun. Math. Phys. 78 (1980), 201-246.

21. (1983), 411-414.

22. J. Miller and B. Derrida, Weak disorder expansion for the Anderson model on a tree, J. Stat. Phys. (1994).

23. A. D. Mirlin and Y. V. Fyodorov, Localization transition in the Anderson model on 
the Bethe lattice: spontaneous symmetry breaking and correlation functions, Nucl. Phys. B 366 (1991), 507-532.

24. L. Nirenberg, Topics in Nonlinear Functional Analysis, Courant Institute of Mathematical Sciences, New York, 1974.

25. L. Pastur, Spectra of random selfadjoint operators, Russ. Math. Surv. 28 (1973), $1-67$.

26. B. Simon and T. Wolff, Singular continuum spectrum under rank one perturbations and localization for random Hamiltonians, Commun. Pure. Appl. Math. 39 (1986), 75-90.

Department of Mathematics, University of California, Irvine, CA 927173875

E-mail address: aklein@math.uci.edu 